\title{
ESTADO DEL ARTE: MÉTRICAS DE CALIDAD PARA EL DESARROLLO DE APLICACIONES WEB
}

\section{STATE OF ART: QUALITY METRICS FOR WEB APPLICATION DEVELOPMENT}

\author{
Fausto Fabián Redrován Castillo ${ }^{1}$ \\ Nancy Magaly Loja Mora² \\ Kevin David Correa Elizaldes ${ }^{3}$ \\ Josias Israel Piña Orozco ${ }^{4}$
}

1. Universidad Técnica de Machala. Grupo de Investigación de la carrera de Ingeniería de Sistemas (Ecuador). E-mail: fredrovan@utmachala.edu.ec

2. Universidad Técnica de Machala. Grupo de Investigación de la carrera de Ingeniería de Sistemas (Ecuador).E-mail: nmloja@utmachala.edu.ec

3. Universidad Técnica de Machala. Grupo de Investigación de la carrera de Ingeniería de Sistemas (Ecuador). E-mail: kdcorrea est@tmachala.edu.ec

4. Universidad Técnica de Machala. Grupo de Investigación de la carrera de Ingeniería de Sistemas (Ecuador). E-mail: jipina est@utmachala.edu.ec

\section{Citación sugerida:}

Redrován Castillo, F.F., Loja Mora, N.M., Correa Elizaldes, K.D. y Piña Orozco, J.I. (2017). Estado del arte: Métricas de calidad para el desarrollo de aplicaciones web. 3C Tecnología: glosas de innovación aplicadas a la pyme, 6(4). 1-12. DOI: <http://dx.doi.org/10.17993/3ctecno.2017.v6n4e24.1-12/>. 


\section{RESUMEN}

Las aplicaciones Web ubicadas en los servidores y accesibles por medio de internet o intranet, son sensibles a diferentes métricas de calidad. Estas métricas de calidad pueden ser la seguridad, el grado de cobertura, tiempos de respuesta, disponibilidad, etc. Debido al gran avance de este tipo de software existen varios modelos actuales que permiten elaborar un control de calidad de las cuales se puede resaltar la ISO/IEC 9126 y ISO/IEC 25000. El objetivo del presente trabajo es analizar los diferentes modelos y estándares de calidad orientados al producto de software, mediante recopilación de datos bibliográficos para la identificación de las métricas eficaces en el desarrollo de aplicaciones Web. En la investigación se demostró que la calidad de estas aplicaciones se ven afectadas por la cantidad de métricas que puedan cumplirse, además se evidenció que no existe un método que se dedique específicamente a las aplicaciones Web.

\section{ABSTRACT}

Web applications located on servers and accessible via internet or intranet, are sensitive to different quality metrics. These quality metrics can be security, degree of coverage, response times, availability, etc. Due to the great advance of this type of software, there are several current models that allow to elaborate a quality control of which the ISO / IEC 9126 and ISO / IEC 25000 can be highlighted. The objective of the present work is to analyze the different models and standards of quality oriented to the software product, by collecting bibliographic data for the identification of the effective metrics in the development of Web applications. The research showed that the quality of these applications is affected by the number of metrics that can be met, in addition it was evidenced that there is no method that is dedicated specifically to Web applications.

\section{PALABRAS CLAVE}

Métricas de calidad, aplicaciones Web, normas estándar.

\section{KEY WORDS}

Metric quality, Web applications, standards. 


\section{INTRODUCCIÓN}

Desarrollar una aplicación Web de calidad es una tarea imprescindible para todas aquellas empresas que desean brindar servicios a través de la Web. Por lo tanto, medir su calidad es de vital importancia para las organizaciones que pretenden posicionarse en la cima del Internet.

Las aplicaciones Web ofrecidas por los programadores independientes pueden ofrecer funcionalidad, pero estas no están sujetas a rigurosos controles de calidad que proporcionan las empresas de desarrollo con muchos años de experiencia y que consten con debidas certificaciones que aprueben su calidad como empresa y los productos que llevan al mercado.

La calidad según [1] se puede definir como la totalidad de características de una entidad que refieren su capacidad de satisfacer las necesidades implícitas y explícitas del usuario, por otro lado Pressman [2] se refiere a la calidad del software como "la concordancia con los requerimientos funcionales $\mathrm{y}$ de rendimiento explícitamente establecidos, con los estándares de desarrollo explícitamente documentados y con las características implícitas que se espera de todo software desarrollado profesionalmente".

Los modelos de calidad surgen para describir las características del software, sus relaciones, cómo pueden ser medidas y la forma en la que las mediciones pueden ser interpretadas [3]; pero cómo saber cuál de todas proporciona métricas precisas para productos de software, esto se debe a la cantidad de métricas cumplidas en los diferentes métodos de evaluación. La realidad actual de las empresas de desarrollo y los softwares que son creados día a día demandan la incorporación de normas que certifiquen un avalúo de calidad. Los productos y procesos que se llevan a cabo en las empresas están vinculados estrechamente, ya que el proceso que se tome en la realización del software permitirá un resultado de calidad.

Los modelos, estándares y normas que se encuentran relacionados con la empresa, el desarrollo y producto de software son muchos, de cuales se pueden dividir en tres grupos, calidad del producto, la calidad del proceso y la calidad de la empresa.

Las diferentes formas de medir la calidad son por medio de una serie de métricas específicas orientadas a los productos de software, aunque no están especificados para dichas aplicaciones. Por ello, en el siguiente documento se tiene como objetivo analizar los diferentes modelos y estándares de calidad orientados al producto de software, mediante recopilación de datos bibliográficos para la identificación de las métricas más eficaces en el desarrollo de aplicaciones Web. 


\section{ANTECEDENTES O ESTADO DEL ARTE}

\subsection{APLICACIONES WEB}

Tabla 1. Estado del arte - Aplicaciones web.

\begin{tabular}{|c|l|l|}
\hline Año & Título del Libro & \multicolumn{1}{c|}{ Contenido } \\
\hline 2010 & $\begin{array}{l}\text { Desarrollo } \\
\text { Profesional de de } \\
\text { Aplicaciones Web }\end{array}$ & $\begin{array}{l}\text { "La característica común de todas las aplicaciones Web es el } \\
\text { hecho de centralizar el software para facilitar las tareas de } \\
\text { mantenimiento y actualización de grandes sistemas" [4] } \\
\text { "Las aplicaciones web son aplicaciones q las que se accede } \\
\text { mediante un navegador y están alojadas en servidores dentro } \\
\text { de una Intranet o en Internet." [5] }\end{array}$ \\
\hline Aplicaciones Web & &
\end{tabular}

Fuente: 1 [4], [5].

De acuerdo a los autores las aplicaciones web funcionan exclusivamente con conexión a internet permitiendo al usuario interactuar y mantener una comunicación entre sí. Estas aplicaciones contienen una sección administrable donde el responsable del sistema podrá realizar modificaciones con el aspecto o presentación de la misma.

\subsection{MÉTRICAS DE CALIDAD DEL SOFTWARE}

Tabla 2. Estado de arte - Métricas de calidad del software.

\begin{tabular}{|c|l|l|}
\hline Año & \multicolumn{1}{|c|}{ Título Del Libro } & \multicolumn{1}{c|}{ Contenido } \\
\hline \multirow{2}{*}{2008} & $\begin{array}{l}\text { Métricas aplicadas a los } \\
\text { modelos de calidad: caso de } \\
\text { uso en los SIG }\end{array}$ & $\begin{array}{l}\text { La calidad consiste en aquellas características de producto que } \\
\text { se basan en las necesidades del cliente y que por eso brindan } \\
\text { satisfacción con el producto. [9] }\end{array}$ \\
\hline 2009 & $\begin{array}{l}\text { Métricas de Calidad de los } \\
\text { Sistemas de Información }\end{array}$ & $\begin{array}{l}\text { Las métricas son escalas de unidades sobre las cuales puede } \\
\text { medirse un atributo cuantificable. [6] }\end{array}$ \\
\hline
\end{tabular}

Fuente: 2 [6] [9].

El uso de las métricas establece cómo se debe ajustar el software a los requisitos implícitos y explícitos del cliente. Es decir, la medición para que el sistema se adapte a los requisitos establecidos, las métricas de calidad de sistemas de información utilizados para evaluar y controlar el proceso de desarrollo del software, deben permitir:



Figura 3. Proceso para la evaluación de calidad. 


\subsection{ESTÁNDARES DE CALIDAD A NIVEL DE PROCESO}

Tabla 3. Factores de calidad a nivel de proceso en el modelo MOSCA.

\begin{tabular}{|c|c|c|}
\hline \multicolumn{3}{|c|}{ Modelo de Calidad MOSCA } \\
\hline Categoría & & sticas \\
\hline Soporte & $\begin{array}{l}\text {-Aseguramiento de la Calidad } \\
\text {-Revisión Conjunta } \\
\text {-Auditoria } \\
\text {-Resolución de Problemas }\end{array}$ & $\begin{array}{l}\text {-Documentación } \\
\text {-Verificación } \\
\text {-Validación } \\
\text {-Resolución de Problemas }\end{array}$ \\
\hline Gestión & $\begin{array}{l}\text {-Gestión } \\
\text {-Gestión de Calidad } \\
\text {-Gestión del Riesgo }\end{array}$ & $\begin{array}{l}\text {-Gestión } \\
\text {-Gestión de Proyecto } \\
\text {-Gestión de Calidad } \\
\text {-Gestión del Riesgo }\end{array}$ \\
\hline Organizacional & $\begin{array}{l}\text {-Gestión de Cambio } \\
\text {-Mejoramiento del Proceso } \\
\text {-Medición } \\
\text {-Reúso }\end{array}$ & $\begin{array}{l}\text {-Establecimiento del Proceso } \\
\text {-Evaluación del Proceso } \\
\text {-Mejoramiento del Proceso } \\
\text {-Infraestructura }\end{array}$ \\
\hline
\end{tabular}

Fuente: [11].

Mosca, considera que "No existe calidad del producto sin la calidad en el proceso y viceversa", contempla como punto principal el analizar la empresa en sus etapas de desarrollo. Este modelo garantiza el balance entre la eficiencia y la efectividad del proceso de desarrollo a través de una propuesta equilibrada de prácticas bases.

\subsection{MODELOS Y ESTÁNDARES DE CALIDAD A NIVEL DE PRODUCTO}

Tabla 4. Modelos de calidad a nivel de productos.

\begin{tabular}{|c|c|c|}
\hline \multicolumn{3}{|c|}{ MODELO } \\
\hline NOMBRE & CARACTERISTICA & FACTORES DE CALIDAD \\
\hline FURPS & $\begin{array}{l}\text { "Una limitación de este modelo de } \\
\text { calidad es que no tiene en cuenta la } \\
\text { portabilidad de los softwares, factor } \\
\text { digno de consideración en función de las } \\
\text { exigencias actuales que recaen sobre el } \\
\text { proceso de desarrollo." [12] }\end{array}$ & $\begin{array}{l}\text {-Funcionalidad } \\
\text {-Usabilidad } \\
\text {-Confiabilidad } \\
\text {-Soporte }\end{array}$ \\
\hline DROMEY & $\begin{array}{l}\text { Este modelo describe la idea de } \\
\text { relacionar atributos del producto con } \\
\text { atributos de calidad para su evaluación. } \\
\text { [12] }\end{array}$ & $\begin{array}{l}\text {-Corrección } \\
\text {-Estructuración } \\
\text {-Modularidad } \\
\text {-Descriptivo }\end{array}$ \\
\hline WebQEM & $\begin{array}{l}\text { Emplea un modelo de calidad que } \\
\text { proporciona un enfoque cuantitativo y } \\
\text { sistemático para evaluar y compara } \\
\text { productos web en la fase operativa y en } \\
\text { la fase de desarrollo de ciclo de vida del } \\
\text { producto [13] }\end{array}$ & $\begin{array}{l}\text {-Usabilidad } \\
\text {-Funcionalidad } \\
\text {-Fiabilidad } \\
\text {-Eficiencia }\end{array}$ \\
\hline
\end{tabular}

Fuente: autores.

Los diversos modelos que existen para medir y regular la calidad a nivel de producto ofrecen una serie de características comunes a las ISO/IEC 9126, debido a que este posee gran acogida en el campo informático. Otros como el DROMEY establecen sus propias características para la evaluación teniendo en cuenta la capacidad de ser modificado el producto luego de ser implementado. 


\subsection{MODELOS Y ESTÁNDARES DE CALIDAD DEL SOFTWARE A NIVEL DE PROCESOS}

Tabla 5. Modelos y estándar de calidad a nivel de procesos.

\begin{tabular}{|c|c|}
\hline Modelo o Estándar. & Definición \\
\hline CMMI [14] & $\begin{array}{l}\text {-Planificación de proyectos } \\
\text {-Monitoreo y Control de Proyectos } \\
\text {-Gestión Integrada de Proyectos (IPPD) } \\
\text {-Gestión Integrada de Proveedores (SS) } \\
\text {-Gestión de Riesgos Gestión de Proyectos Cuantitativos } \\
\text {-Análisis y Análisis Causal de Medición y Análisis } \\
\text {-Análisis y resolución de decisiones } \\
\text {-Integración de productos } \\
\text {-Verificación } \\
\text {-Enfoque del proceso organizacional } \\
\text {-Innovación e implementación organizacional. }\end{array}$ \\
\hline ISO/IEC 15504 (SPICE) [15] & $\begin{array}{l}\text {-Proceso de Suministro } \\
\text {-Proceso de Gestión del Modelo de Ciclo de Vida } \\
\text {-Proceso de Planificación del Proyecto } \\
\text {-Proceso de Evaluación y Control del Proyecto } \\
\text {-Proceso de Medición } \\
\text {-Proceso de Análisis delos Requisitos del Sistema } \\
\text {-Proceso de Aseguramiento de la Calidad del Software }\end{array}$ \\
\hline ISO/IEC 12207 [16] & $\begin{array}{l}\text {-Documentación } \\
\text {-Gestión de la configuración. } \\
\text {-Aseguramiento de calidad. } \\
\text {-Resolución de problemas. } \\
\text {-Infraestructura. } \\
\text {-Mejora. } \\
\text {-Recursos Humanos. }\end{array}$ \\
\hline ISO/IEC 29110 [17] & $\begin{array}{l}\text {-Proceso de planificación del proyecto } \\
\text {-Evaluación de proyectos y Control del proceso } \\
\text {-Proceso de análisis de requisitos software } \\
\text {-Proceso de gestión de riesgo } \\
\text {-Proceso de aseguramiento de calidad de software. }\end{array}$ \\
\hline РМВоОК [18] & $\begin{array}{l}\text {-Gestión de la Integración del Proyecto } \\
\text {-Gestión del Alcance del Proyecto } \\
\text {-Gestión de Tiempo del Proyecto } \\
\text {-Gestión de los Costos del Proyecto } \\
\text {-Gestión de la Calidad del Proyecto } \\
\text {-Gestión de Recursos Humanos del Proyecto } \\
\text {-Gestión de las Comunicaciones del Proyecto } \\
\text {-Gestión de los riesgos del Proyecto }\end{array}$ \\
\hline ISO 9001 [19] & $\begin{array}{l}\text {-Alcance } \\
\text {-Referencias normativas } \\
\text {-Términos y definiciones } \\
\text {-Contexto de la organización } \\
\text {-Liderazgo } \\
\text {-Planificación } \\
\text {-Evaluación del desempeño }\end{array}$ \\
\hline COBIT $4.0[20]$ & $\begin{array}{l}\text {-Confiabilidad } \\
\text {-Escalabilidad } \\
\text {-Capacitación } \\
\text {-Flexibilidad } \\
\text {-Tiempo Requerido } \\
\text {-Componentes O Dominios } \\
\text {-Comunicación Interna } \\
\text {-Responsabilidad Por El Sistema De Ci Evaluado } \\
\text {-Enfoque }\end{array}$ \\
\hline ITIL [21] & $\begin{array}{l}\text {-Gestión de Incidencias } \\
\text {-Gestión de la Configuración }\end{array}$ \\
\hline
\end{tabular}






Fuente: autores.

Se puede evidenciar que en la actualidad se encuentran muchos estándares para evaluar los procesos de desarrollo de software. Destaca la PMBOOK como la más popular entre los ingenieros de desarrollo, pues especifica todas las etapas de planificación y control en la creación de un software.

\section{METODOLOGÍA}

La investigación a realizarse es de tipo heurística, hermenéutica y descriptiva con el fin de extraer de las unidades de análisis del material documental, los datos pertinentes y someterlos a un proceso de revisión, reseña y descripción.

Para realizar el siguiente trabajo se han denominado diferentes etapas que se centrarán en la evaluación de calidad de las metodologías en aplicaciones Web, las cuales son:

1. Investigación bibliográfica de trabajos que correspondan con estudios similares.

2. Determinación y procesamiento de datos recopilados de las diferentes normas estándares.

3. Establecimiento de análisis comparativo por las diferentes métricas.

4. Análisis de los datos obtenidos y verificación de los mismos.

5. Elaboración de los resultados, discusión y la conclusión sobre la temática propuesta.

\section{RESULTADOS}

Las aplicaciones basadas en la Web a través del tiempo han requerido cumplir con muchos criterios de calidad y esto se puede evidenciar en trabajos e investigaciones realizadas por diferentes autores:

Tabla 6. Revisión de la literatura sobre calidad Web.

\begin{tabular}{|l|l|}
\hline \multicolumn{1}{|c|}{ INVESTIGACIONES } & \multicolumn{1}{c|}{ FACTORES CONSIDERADOS } \\
\hline Barron. [23] & Gráficos, Texto, Vínculos, Tamaño del sitio, Longitud, Multimedia \\
\hline Olsina [24] & Funcionalidad, Usabilidad, Eficiencia, Fiabilidad del sitio. \\
\hline Liu y Arnett [25] & $\begin{array}{l}\text { Capacidad de aprendizaje, Diversión, Calidad del sistema, Uso del } \\
\text { sistema. }\end{array}$ \\
\hline Buenadicha [26] & Accesibilidad, Velocidad, Navegabilidad y Contenido. \\
\hline Aladwani y Palvia [27] & $\begin{array}{l}\text { Contenido específico, Calidad de contenido, Adecuación tecnológica, } \\
\text { Apariencia. }\end{array}$ \\
\hline
\end{tabular}

Fuente: autores. 
Al analizar cada uno de los trabajos se han encontrado diferencias significativas en los aspectos importantes que debe contener una aplicación Web, lo que representa un problema pues no se encuentran normas establecidas aplicadas. Esto lleva a creer que, a diferencia de las aplicaciones de escritorio, las páginas de Internet contemplan aspectos variantes.

A continuación, se muestra una tabla 7 , donde se relacionan las normas de calidad con las métricas que presentan dichos modelos, cabe recalcar que estas métricas no están enfocadas específicamente a las aplicaciones Web:

Tabla 7. Comparativa de las métricas entre las normas, estándares y métodos de calidad.

\begin{tabular}{|c|c|c|c|c|c|c|}
\hline Características de calidad & 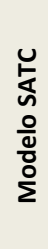 & 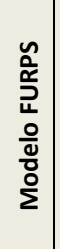 & 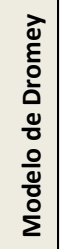 & 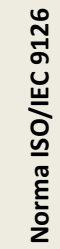 & 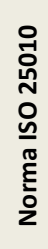 & $\begin{array}{l}\overline{\widetilde{T}} \\
\text { 。 }\end{array}$ \\
\hline Facilidad de uso & & $\mathrm{x}$ & $\mathrm{x}$ & $\mathrm{x}$ & $\mathrm{x}$ & 5 \\
\hline Integridad & $x$ & & & & & 2 \\
\hline Corrección & $\mathrm{x}$ & & & & & 2 \\
\hline Confiabilidad & & $x$ & $\mathrm{x}$ & $\mathrm{x}$ & $\mathrm{x}$ & 6 \\
\hline Eficiencia & & & $x$ & $\mathrm{x}$ & $\mathrm{x}$ & 5 \\
\hline Facilidad de mantenimiento & $x$ & & $\mathrm{x}$ & $x$ & $x$ & 6 \\
\hline Facilidad de prueba & & & & & & 2 \\
\hline Portabilidad & & & $x$ & $\mathrm{x}$ & $\mathrm{x}$ & 5 \\
\hline Ingeniería Humana & & & & & & 1 \\
\hline Fácil de entender & $\mathrm{x}$ & & & & & 2 \\
\hline Funcionalidad & & $x$ & $x$ & $\mathrm{x}$ & $\mathrm{x}$ & 5 \\
\hline Facilidad del soporte & & $x$ & & & & 1 \\
\hline Trazabilidad & $x$ & & & & & 1 \\
\hline Documentación & $x$ & & & & & 1 \\
\hline Conformidad & & & & & & 1 \\
\hline
\end{tabular}

Fuente: autores.

Se puede observar que las características más relevantes entre todos los modelos son: Facilidad de uso, Confiabilidad, Eficiencia, Facilidad de mantenimiento, Portabilidad y Funcionalidad.

\subsection{MÉTRICAS DE CALIDAD EN LAS APLICACIONES WEB}

En la recopilación bibliográfica se ha evidenciado la concordancia de criterios entre los diversos autores, normas, estándares y los modelos de calidad. Con lo cual, se han especificado como características comunes de calidad las siguientes categorías: contenido, seguridad, navegabilidad, usabilidad y diseño.

La navegabilidad es un aspecto importante para la calidad, la cual ayuda al usuario a encontrar la información requerida a través de palabras clave o un menú permanente.

El contenido otorga al usuario conocimiento de una empresa. Por ello, este aspecto puede abarcar un gran potencial de calidad al ofrecer confianza de la reputación y de los productos o servicios prestados por la compañía. 
El diseño es un factor de calidad relativamente importante, las aplicaciones web tienen mayor cabida en el medio cuando esta consta con un aspecto visual apreciado por la sociedad que contengan colores e interfaz amigable.

La usabilidad define la interacción del usuario con la aplicación Web, centrándose en la facilidad de navegación y rendimiento de las tareas.

Con respecto a la seguridad esta representa la capacidad del producto software para lograr prevenir el acceso no autorizado.

\subsection{CASO DE ESTUDIO}

\section{- Selección de un área piloto de estudio}

La investigación de campo está enfocada en la ciudad de Machala, provincia de El Oro. Actualmente en esta ciudad las empresas dedicadas al desarrollo de software son cinco; en base a esto se afirma que la población es pequeña y no se necesita utilizar cálculos muéstrales.

\section{- Análisis estadístico}

En este punto se procedió a recolectar de manera sistemática los datos mediante la aplicación de las entrevistas y encuestas con preguntas claves que están directamente relacionadas con las métricas de calidad, luego de eso se procedió a tabular los resultados obtenidos.

- Calidad web aplicada en la ciudad de Machala

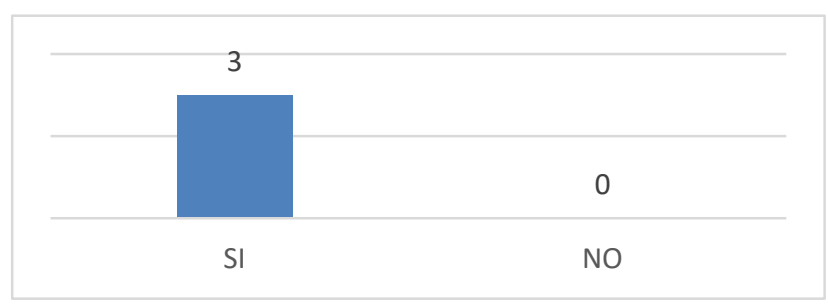

Gráfico 1. Empresas que aplican métricas de calidad.

Se pudo identificar que tres empresas aplican algún tipo de control de calidad, lo cual propone que en la mayoría de la ciudad los Softwares ofrecen calidad en sus productos Web.

- Métricas aplicadas a las empresas de desarrollo

Tabla 8. Características según ISO/EC 9126.

\begin{tabular}{|l|r|r|r|r|r|}
\hline \multirow{2}{*}{\multicolumn{1}{c|}{ Características según ISO/IEC 9126 }} & \multicolumn{3}{c|}{ Empresas } & \multirow{2}{*}{} \\
\cline { 2 - 4 } & E1 & E2 & E3 & \multicolumn{1}{|c|}{ TOTAL } \\
\hline FUNCIONALIDAD & 5 & 5 & 5 & 5,0 \\
\hline FIABLIDAD & 4 & 5 & 5 & 4,7 \\
\hline USABILIDAD & 5 & 5 & 5 & 5,0 \\
\hline EFICIENCIA & 4 & 5 & 5 & 4,7 \\
\hline MANTENIBILIDAD & 4 & 5 & 5 & 4,7 \\
\hline PORTABILIDAD & 1 & 1 & 1 & 1,0 \\
\hline
\end{tabular}

Fuente: autores. 
Se puede observar que esta métrica es eficientemente adoptada para el control del producto Web, siendo las más importantes: la funcionalidad y usabilidad.

Tabla 9. Características según revisión bibliográfica en base a la Web
\begin{tabular}{|c|r|r|r|r|r|}
\hline \multirow{2}{*}{$\begin{array}{c}\text { Características según revisión bibliográfica } \\
\text { en base a la Web }\end{array}$} & \multicolumn{3}{|c|}{ Empresas } & \\
\cline { 2 - 5 } CONTENIDO & E1 & E2 & E3 & TOTAL \\
\hline VISIBILIDAD & 5 & 5 & 5 & 5,0 \\
\hline VELOCIDAD & 4 & 4 & 4 & 4,0 \\
\hline NAVEGABILIDAD & 5 & 5 & 5 & 5,0 \\
\hline INTERACTIVIDAD & 5 & 5 & 5 & 5,0 \\
\hline DISEÑO & 5 & 5 & 4 & 4,7 \\
\hline ACCESIBILIDAD & 5 & 5 & 4 & 4,7 \\
\hline & 4 & 4 & 5 & 4,3 \\
\hline
\end{tabular}

Fuente: autores.

Las características analizadas, recopiladas por los trabajos de diferentes investigaciones sobre la calidad en las aplicaciones Web son eficientemente adoptadas para el control del producto Web, siendo las más importantes: el contenido, la velocidad y la navegabilidad.

\section{DISCUSIÓN Y CONCLUSIONES}

Actualmente, las empresas tienen la urgencia de ser conocidas por la sociedad y el medio informático, por ello las ofrecen aplicaciones Web de calidad, siendo la respuesta óptima para dar a conocer internacionalmente una organización que ofrece servicios o productos.

La calidad de las aplicaciones basadas en la Web es importante, puesto que al proveer de una mejor calidad tendrá una mayor probabilidad de adquirir éxito en su negocio. Las funcionalidades que ofrecen las normas ISO no son dirigidas tanto a las aplicaciones Web como a las de escritorio, es así como se vio en necesidad de identificar las características específicas para medir dicha calidad.

Dado la investigación y los resultados obtenidos, se puede concluir que:

Existen diversos modelos y normas que establecen métricas de calidad para la evaluación del producto, sin embargo, en la actualidad se considera factible aplicar la norma ISO/IEC 25000, debido a que abarca la norma ISO/IEC 9126, que describe las particularidades de un modelo de calidad del producto de software. 


\section{REFERENCIAS BIBLIOGRÁFICAS}

[1] N. Bevan, Quality in use: Meeting user needs for quality, Journal of Systems and Software, 1999.

[2] R. Pressman, Ingeniería del Software. Un enfoque práctico, Madrid: McGraw-Hill Interamericana, 2002.

[3] J. Córdoba, "Modelo de Calidad para Portales Bancarios," [En línea]. Available: http://www.dlsi.ua.es/ ccachero/papers/clei07.pdf. [Último acceso: 201610 31].

[4] F. Berzal y J. F. Cortijo, Desarrollo Profesional de Aplicaciones Web con ASP.NET, México: iKor Consulting, 2010.

[5] J. Niño, Aplicaciones web, Madrid: Editex S. A., 2010.

[6] W. Siabato, Métricas aplicadas a los modelos de calidad: caso de uso en los SIG, 2008.

[7] R. Pérez, Modelo de especificación de calidad para sitios web universitarios (MOSCAWEB), Valencia: Universidad Nacional Abierta, 2007.

[8] H. Solano y I. Torres, Análisis de Frameworks para eldesarrollo de aplicaciones móviles en la plataforma Android, Cuenca: Universidad del Azuay, 2013.

[9] A. Belcastro, Requerimientos de calidad de sitios web destinado a servicios de catering, UNPSJB, 2014.

[10] M. Beth, M. Konrad y S. Shrum, CMMI, Pearson Educación, 2009.

[11] A. Alarcón y J. González, Guía para pyes desarrolladoras de software, basada en la norma ISO/IEC 15504, 2011.

[12] A. Gallegos y P. Ortiz, Elaboración del estándar de aplicación de la norma ISO/IEC 12207, al desarrollo de aplicaciones de software para la Utic de la ESPE, 2011.

[13] ISO, ISO/IEC 29110-4-1, 2011.

[14] Project Management Institute, Inc., Guía de los fundamentos para la dirección de proyectos, 2013.

[15] ISO, ISO 9001: 2015, 2015.

[16] D. Ibañez, Implantación de directrices ITIL en un Departamento de Soporte y Operaciones de una empresa, 2012.

[17] ITIL Foundation, «ITIL V3,» [En línea]. Available: http://itilv3.osiatis.es/itil.php.

[18] AENOR, ISO/IEC 20000 Guía completa de aplicación para la gestión de los servicios de tecnologías de la información, Madrid, 2009.

[19] A. Barrom, B. Tompkins y D. Tai, ,Design guidelines for the World Wide Web, 1998.

[20] L. Olsina y G. D. Godoy, Specifying quality characteristics and attributes for websites. First ICSE Worksshop on Web Engeering, 1999.

[21] C. Liu y K. Arnett, Exploring the factor associated with the web site success in the contexto f electronic commerce, 2000.

[22] M. Buenadicha, A. Chamorro y O. González, «A new web assessment index: spanish universities analysis," 2001. 
[23] A. Aladwani y P. Palvia, «Developing and validating an instrument for measuring userperceived web quality,» 2002.

[24] R. M. Caivano y L. N. Villoria, Aplicaciones web 2.0, México: Eduvim, 2009.

[25] S. J. Tomalá, «Métricas de Calidad de los Sistemas de Información - aplicación en la Certificación de Calidad de un Sistema de un empresa del sector hidrocarburífero,» Escuela Superior Politécnica del Litoral, Guayaquil, 2009.

[26] J. H. Corrochano, La calidad del Producto de sotware, Madrid: AtSistemas, 2013.

[27] O. Cuauhtémoc Lemus, "Calidad de software: Procesos, Arquitectura,» Jalisco, 2007. [En línea]. Available: http://www.cimat.mx/.

[28] H. F. Jimenez, Modelos de Ciclo de Vida de Desarrollo de Software, 2002.

[29] J. F. Urbalejo, IMPLEMENTACIÓN Y DEBUGGING: ciclo de vida de software, 2014.

[30] J. H. Corrochano, La calidad del Producto de sotware, Madrid: AtSistemas, 2013.

[31] F. Scalone, Estudio comparativo de los modelos y estandares de calidad del software, Buenos Aires, 2006.

[32] A. De Antonio, GESTIÓN, CONTROL Y GARANTÍA DE LA CALIDAD DEL SOFTWARE, 2005.

[33] A. F. M. Antonieta, Calidad en la Industria del Software. La Norma ISO-9126, 2012.

[34] ISO, «ISO25000,» [En línea]. Available: http://iso25000.com/index.php/normas-iso25000/iso-25010. [Último acceso: 201630 30].

[35] O. L. Londoño Palacio, GUÍA PARA CONSTRUIR ESTADOS DEL ARTE, Bogotá, 2014.

[36] M. Misic y K. Johson, Benchmarking: a tool for web site evaluation and improvement, 1999.

[37] J. Palmer, "Web site usability, design and performance metrics,» 2002.

[38] C. A. García, «GUIA TECNICA PARA EVALUACION DE SOFTWARE,» Saramago, 2005. 